\title{
Construct Validity of the Montreal Cognitive Assessment (MoCA)
}

\author{
Sandra Freitas, ${ }^{1}$ Mário R. Simões, ${ }^{1}$ João Marôco,${ }^{2}$ Lara Alves, ${ }^{3}$ AND Isabel Santana ${ }^{4}$ \\ ${ }^{1}$ Centro de Investigação do Núcleo de Estudos e Intervenção Cognitivo Comportamental (CINEICC), Faculty of Psychology and \\ Educational Sciences, University of Coimbra, Coimbra, Portugal \\ ${ }^{2}$ Psychology and Health Research Unit (UIPES) \& Departamento de Estatística, ISPA - Instituto Universitário, Lisboa, Portugal \\ ${ }^{3}$ Faculty of Psychology and Educational Sciences, University of Coimbra, Coimbra, Portugal \\ ${ }^{4}$ Neurology Department of the Coimbra University Hospital \& Faculty of Medicine, University of Coimbra, Coimbra, Portugal
}

(Received August 15, 2011; Final Revision October 20, 2011; Accepted October 24, 2011)

\begin{abstract}
The Montreal Cognitive Assessment (MoCA) is a brief instrument developed for the screening of milder forms of cognitive impairment. The present study aims to assess the construct related validity of the MoCA through the establishment of the factorial, convergent, and discriminant related validities, and the reliability of data. In a Portuguese sample of 830 participants, several models were tested using Confirmatory Factor Analysis. Although all tested models showed a good fit, the six-factor model based on the conceptual model proposed by the MoCA's authors showed a significantly better fit. The results allowed us to establish the factorial, convergent, and discriminant validity of this six-dimensional structure. An overall psychometric adequacy of the items, and a good reliability were also found. This study contributes to overcome an important gap in the construct related validity of this instrument. The present findings corroborate the six-dimensional structure of the MoCA and provide good evidence of the construct related validity. The MoCA has proved to be an appropriate measure for cognitive screening taking into account different cognitive domains, which will enable clinicians and researchers to use this test and its six latent dimensions to achieve a better understanding of the individuals' cognitive profile. (JINS, 2012, 18, 242-250)
\end{abstract}

Keywords: Cognitive disorders, Alzheimer disease, Neuropsychological test, Psychometrics, Validity and reliability, Factor analysis

\section{INTRODUCTION}

The Montreal Cognitive Assessment (MoCA; Nasreddine et al., 2005) is one of the most recent screening tests, specifically developed for the screening of milder forms of cognitive impairment. Surpassing the limitations of the Mini-Mental State Examination (MMSE; Folstein, Folstein, \& McHugh, 1975), the MoCA is now recognized as one of the best cognitive screening tests (Appels \& Scherder, 2010; Ismail, Rajji, \& Shulman, 2009; Jacova, Kertesz, Blair, Fisk, \& Feldman, 2007; Lonie, Tierney, \& Ebmeier, 2009). Although it was initially designed to assess the cognitive state of patients with Mild Cognitive Impairment and with Alzheimer's disease, currently the MoCA is an extensively validated screening tool for many disorders. Several studies have proved the good psychometric properties and excellent sensitivity of data

Correspondence and reprint requests to: Sandra Freitas, Faculty of Psychology and Educational Sciences, University of Coimbra, Rua do Colégio Novo, Apartado 6153, 3001-802 Coimbra, Portugal. E-mail: sandrafreitas 0209@gmail.com gathered with the MoCA. Its ability for the early identification of cognitive decline throughout the course of the disease, has promoted its rapid international dissemination (see studies in http://www.mocatest.org). Beyond its screening properties, the MoCA can also be used as a quantitative estimate of the overall cognitive ability (Koski, Xie, \& Finch, 2009; Koski, Xie, \& Konsztowicz, 2011), and as an indicator of the global cognitive decline in patients' longitudinal monitoring (Freitas, Santana, \& Simões, 2010).

Despite its current widespread use and clinical validity studies, the internal structure of the MoCA needs to be studied more systematically. Nasreddine et al. (2005) made a theoretical proposal according to which the MoCA's 30 items could be categorized into the following cognitive domains: executive functions; visuospatial abilities; short-term memory; language; attention, concentration, and working memory; and temporal and spatial orientation.

Duro, Simões, Ponciano, and Santana (2010) analyzed the dimensions underlying the MoCA in a heterogeneous clinical sample with cognitive decline, not having confirmed the 
structure originally suggested by the MoCA authors (Nasreddine et al., 2005). In this study, a two-factor model was found as best solution: the first factor was designated "Memory," it comprised memory, language and orientation tasks; the second factor was designated "Attention/Executive Functions," it included attention, executive functions and visuospatial skills. It should be noted that this study used the MoCA's Portuguese experimental version, while the present study uses the MoCA's Portuguese final version, which resulted from the adaptation process for the Portuguese population and which is significantly different from the first. The main changes occurred at the level of the words of the memory task and sentences for repetition (translated version used in MoCA's Portuguese experimental version was not equivalent with the original version in words and sentences length), as well as in administration and scoring instruction manual (which was improved after the studies conducted with the experimental version), as described by Freitas, Simões et al. (2010). To date, and as far as we know, there have been no other studies on the factorial structure of the MoCA in clinical populations and there are not any studies with community samples, which represents an important gap in the construct related validity of this instrument.

Translation, adaptation, and validation studies, as well as normative study of the MoCA on the Portuguese population were performed by our research group (Freitas, Santana, et al., 2010; Freitas, Simões, et al., 2010; Freitas, Simões, Alves, \& Santana, 2011; Simões et al., 2008). The present study was undertaken to assess the construct related validity of the MoCA. First, we examined the psychometric properties of the items and scale, and then we analyzed the factorial structure of the MoCA and established the respective factorial, convergent, and discriminant related validities.

\section{METHODS}

\section{Participants and Procedures}

The total sample was composed of 830 participants distributed between two main sub-groups:

Healthy Group

The healthy group was composed by cognitively healthy community dwellers, living in all geographic regions of the Portuguese continental territory. These individuals were recruited through the national health and social security services. This sample served as the basis for the MoCA normative study for the Portuguese population (Freitas et al., 2011). Several demographic and clinical inclusion criteria were considered in the initial selection phase: individuals 25 years and over; Portuguese as the native language and schooling in Portugal; absence of significant motor, visual, or auditory deficits - all of which may influence performance on tests; and to ensure that participants were cognitively healthy adults: autonomy in daily activities; no history of alcoholism or substance abuse; absence of neurological or psychiatric diseases, as well as of chronic unstable systemic disorders with impact in cognition; absence of significant depressive complaints and medication with possible impact in cognition (e.g., psychotropic or psycho-active drugs). The presence of these criteria was confirmed by a psychologist in an interview through a standard questionnaire that included a complete socio-demographic questionnaire, an inventory of current clinical health status, and past habits and medical history. Regarding older participants, this information was also checked with general practitioners, community center directors, and/or an informant, usually an individual that lived with the participant or a close relative. After this initial selection, a set of instruments of global assessment was administered to all subjects: a comprehensive sociodemographic questionnaire; an inventory of the current clinical health status, past habits, and medical history; the MMSE (Folstein et al., 1975; Guerreiro, 1998); the Clinical Dementia Rating scale (CDR; Garret et al., 2008; Hughes, Berg, Danziger, Coben, \& Martin, 1982), only for participants over 49 years old; the Irregular Word Reading Test (TeLPI: Teste de Leitura de Palavras Irregulares; Alves, Simões, \& Martins, 2009), for pre-morbid intelligence estimation; the Subjective Memory Complaints scale (SMC; Ginó et al., 2008; Schmand, Jonker, Hooijer, \& Lindeboom, 1996); and the Geriatric Depression Scale (GDS-30-Barreto, Leuschner, Santos, \& Sobral, 2008; Yesavage et al., 1983). The inclusion criteria required each individual to have a normal performance in the aforementioned instruments. Each participant was assessed in a single session by one of the two psychologists with expertise in neuropsychological assessment. From the initial community-based sample of the 936 volunteers, 194 (20.73\%) were excluded according to data collected in the interview (the most frequent reasons were history of neurological or psychiatric disorder and a history of alcohol abuse), and 92 (9.83\%) were further excluded due to the presence of significant depressive symptoms, or because the performance on the assessment battery was indicative of cognitive impairment according to the Portuguese cutoff points. The final group is composed of 650 cognitively healthy adults that met all the inclusion and exclusion criteria previously described. Cross tabulation of the participants according to socio-demographic variables reproduced the stratification accordingly to age group, gender, educational level, geographic region, geographic localization, and residence area of the Portuguese population (Freitas et al., 2011).

Clinical Group

The clinical group included 90 patients with Mild Cognitive Impairment (MCI) and 90 patients with Alzheimer's disease (AD), recruited from the Dementia Clinic, Neurology Department of Coimbra University Hospital. All patients were clinically examined through neurological, biochemical, and image studies - structural (computed tomography and/or magnetic resonance imaging) and functional (single photon emission computed tomography and/or positron emission tomography) — considered to be essential in the exclusion of other causes and forms of cognitive decline. Furthermore, all patients underwent a comprehensive neuropsychological assessment battery which comprised the following instruments: the MMSE (Folstein et al., 1975; Guerreiro, 1998), the CDR (Hughes et al., 1982; Garret et al., 2008), the Alzheimer's 
Disease Assessment Scale (ADAS; Guerreiro, Fonseca, Barreto, \& Garcia, 2008; Rosen, Mohs, \& Davis, 1984); the TeLPI (Alves et al., 2009), the SMC (Ginó et al., 2008; Schmand et al., 1996), and the GDS-30 (Barreto et al., 2008; Yesavage et al., 1983). Each participant was assessed in a single session by one of two neuropsychologist experts. The diagnoses were previously established through a consensus reached by a multidisciplinary team based on the international criteria for the MCI of the Petersen workgroup (Petersen, 2004) and probable AD (American Psychiatric Association, 2000; McKhann et al., 1984). The MCI group included patients classified as "amnestic MCI" (single or multidomain) (Petersen, 2007) and with a classification of 0.5 on the CDR. The AD group included only patients with mild to moderate severity (classified with $C D R \leq 2$ and MMSE $\geq 12$ points). Only the patients with a completed clinical evaluation, a well established diagnosis according to the above international criteria and a stable clinical condition, without significant comorbidities, were eligible for this study. Thirty patients were initially excluded due to the fact that the differential diagnosis had not yet been established by the multidisciplinary team. Also at the outset the exclusion criteria were: high dementia's severity, recent pharmacotherapy changes, recent psychiatric comorbidity (a clinical diagnosis in the 6 months before the current neuropsychological evaluation), and significant motor, visual or auditory deficits, all of which may influence in neuropsychological assessment. Additionally, each patient who participated in the study had a diagnosis confirmed by a neurologist at the time of the data collection.

For this study purposes, two control sub-groups were selected from the healthy group to match each patient on variables that were found to be predictive of the MoCA's performance (educational level and age) (Freitas et al., 2011). These group's subjects were additionally matched on gender. As a result, a perfect match was obtained between the group with MCI and the control group (afterward named C-MCI group) and between the group with $\mathrm{AD}$ and the associated control group (C-AD Group).

An informed consent was obtained from all the participants after the research aims and procedures, as well as the confidentiality requirements, were fully explained by a member of the research team. For the AD patients who were not capable of providing the informed consent, a legal representative fulfilled that requirement on their behalf. The present research complies with the ethical guidelines on human experimentation stated in the Declaration of Helsinki and was approved by the Portuguese Foundation for Science and Technology and by the Faculty of Psychology and Educational Sciences Scientific Committee.

\section{MEASURE: MONTREAL COGNITIVE ASSESSMENT}

The MoCA is a brief cognitive screening tool, which provides a quick indication of an individual's global cognitive state. It is a tool in paper-and-pencil format, composed by a one-page test, which requires a short administration time, and by a manual explicitly describing the instructions for administering the tasks and objectively portraying the defined scoring system. The MoCA covers a wide range of cognitive functions such as short-term memory, executive functions, visuospatial abilities, language, attention, concentration, working memory, and temporal and spatial orientation. The short-term memory ( 5 points) is tested by a delayed recall of the five nouns previously learned in two trials. The executive functions (4 points) are evaluated by an alternation task adapted from the Trail Making B task, a phonemic fluency task, and a two verbal abstraction tasks. The visuospatial skills (4 points) are assessed using a three-dimensional cube copy and a clock-drawing task (contour, numbers, and hands). The language ( 5 points) is tested by naming three low-familiarity animals, repeating exactly two syntactically complex sentences, and by the phonemic fluency task above mentioned. The attention, concentration, and working memory (6 points) are assessed using digits forward and backward, a sustained attention task, and a serial subtraction task. Finally, the time orientation (4 points) is tested by asking the subject the date, month, year, and day; while the space orientation ( 2 points) is tested by asking the subject in what place and city they are in (Nasreddine et al., 2005). A MoCA score is derived by adding the points of each successfully completed task, in a range from 0 to 30 points, with higher scores indicating better cognitive performance.

\section{Statistical Analyses}

Statistical analyses were performed using the Statistical Package for the Social Sciences (SPSS, version 19.0) (IBM SPSS, Chicago, IL) and the package Analysis of Moment Structures (AMOS version 19.0) (IBM SPSS). Descriptive statistics were used for the sample's characterization and analysis of the distribution responses to each item. The $\chi^{2}$ test and the two-sample t-test allowed the establishment of the two groups comparisons. To assess item/domain discriminating power, the Pearson's correlation coefficient was performed between each item and the total score, each item and cognitive domains, and between each cognitive domain and the MoCA total score (Himmelfarb, 1993). Non-significant correlation coefficients indicate lack of factorial validity, while significant correlation coefficients are an indicator of factorial validity. The Cronbach's alpha is considered as an index of internal consistency. This reliability value should be equal to or higher than 0.7 (Hair, William, Barry, Rolph, \& Ronald, 2010).

The MoCA's diagnostic accuracy for MCI and AD patients was assessed through the receiver operating characteristics (ROC) curve analysis. In this analysis, the areas under the curve (AUC) can vary between 0.5 and 1, with larger AUC indicating better diagnostic accuracy. The optimal cutoff points were calculated for each group according to the highest Youden index, with higher Youden index indicating maximization of the sensibility and specificity.

Confirmatory Factor Analysis (CFA) was conducted to provide further evidence to MoCA's construct validity. Since MoCA's items are dichotomic, model estimation was 
Table 1. Descriptive statistics for the total sample and subgroups

\begin{tabular}{lcccrr}
\hline \hline & $n$ & Education & Age & Gender & MoCA \\
\hline Total sample & 830 & $7.77 \pm 4.699$ & $59.43 \pm 15.498$ & $515(62.0)$ & $408(62.8)$ \\
Healthy group & 650 & $8.16 \pm 4.724$ & $55.84 \pm 15.120$ & $107(59.4)$ & $24.70 \pm 3.668$ \\
Clinical group & 180 & $6.37 \pm 4.338$ & $72.37 \pm 8.270$ & $214(59.4)$ & $14.18 \pm 5.851$ \\
Paired sample & 360 & $6.38 \pm 4.316$ & $71.86 \pm 7.895$ & $55(61.1)$ & $18.59 \pm 6.503$ \\
MCI & 90 & $6.50 \pm 4.565$ & $70.52 \pm 7.950$ & $55(61.1)$ & $18.31 \pm 3.868$ \\
C-MCI & 90 & $6.53 \pm 4.498$ & $69.59 \pm 7.053$ & $52(57.8)$ & $23.64 \pm 3.223$ \\
AD & 90 & $6.23 \pm 4.119$ & $74.22 \pm 8.212$ & $52(57.8)$ & $10.06 \pm 4.410$ \\
C-AD & 90 & $6.24 \pm 4.128$ & $73.10 \pm 7.539$ & $22.33 \pm 3.471$ \\
\hline \hline
\end{tabular}

Note. Healthy group: all cognitively health participants; Clinical group: all patients with MCI and AD; Control Group: cognitively health participants paired with patients; Paired sample: sum of all the patients and their matched controls. Gender is characterized by female's $n$ and respective percentage (\%). Data of others variables are presented as mean \pm standard deviation.

$\mathrm{MoCA}=$ Montreal Cognitive Assessment (maximum score $=30$ ); $\mathrm{MCI}=$ Mild cognitive impairment patients; $\mathrm{C}-\mathrm{MCI}=$ subgroup of controls matched with MCI patients; $\mathrm{AD}=$ Alzheimer's disease patients; $\mathrm{C}-\mathrm{AD}=$ subgroup of controls matched with $\mathrm{AD}$ patients.

done with tetrachoric correlations and the weighted least squares mean and variance adjusted estimation procedure implemented in Mplus6 (Muthén \& Muthén, Los Angeles, CA). To evaluate the goodness of fit of the tested factorial structures the indices $\chi^{2} / d f$, CFI (Comparative Fit Index), TLI (Tucker-Lewis Index), RMSEA (Root Mean Square Error of Approximation) were used. Values indicative of good fit were those generally assumed in CFA (Byrne, 2010; Marôco, 2010): $\chi^{2} / \mathrm{df} \sim 2-3$, CFI, and TLI $>0.9$; and RMSEA $<.05$.

\section{RESULTS}

\section{Sample Characterization}

The total sample was comprised of 830 participants. The characterization of the study sample and in more detail of the all sub-groups is presented in Table 1. For this description were considered the following variables: sample size, educational level, age, gender, and MoCA score.

The control participants included in the paired sub-sample were select from the health group to match in educational level, age and gender to patients of clinical groups. No statistically significant differences were found on the educational level $(t(178)=.049 ; p=.961)$, age $(t(178)=.833 ; p=.406)$, and gender $\left(\chi^{2}(1)=.000 ; p=1.0\right)$ between the MCI and the $\mathrm{C}$-MCI group. Likewise, the $\mathrm{AD}$ and the $\mathrm{C}-\mathrm{AD}$ group did not differ on the educational level $(t(178)=.018 ; p=.986)$, age $(t(178)=.955 ; p=.341)$, and gender $\left(\chi^{2}(1)=.000 ; p=1.0\right)$. The MCI group and the $\mathrm{AD}$ group did not differ on educational level $(t(178)=.411 ; p=.681)$ and gender $\left(\chi^{2}(1)=.092\right.$; $p=.761)$, but nevertheless the $\mathrm{AD}$ patients were significantly older than MCI patients $(t(178)=3.071 ; p=.002)$.

\section{Item Analysis}

To evaluate the distribution responses to each item, the response frequencies were computed (dichotomous items: 0 or 1 point). For the purpose of this analysis, we used the paired sample $(n=360)$ to balance the proportion of healthy controls and patients and minimize the possible influence of sociodemographic differences. The results are provided in Table 2.

A close examination of these results reveals that the items Contour, Lion, Place and City have a high hit rate

Table 2. Analysis of the distribution responses of the MoCA's items

\begin{tabular}{lcc}
\hline \hline Item code & \% Incorrect answers & \% Correct answers \\
\hline TMT-B (adapted) & 56.7 & 43.3 \\
Cube & 62.2 & 37.8 \\
Contour & 2.2 & 97.8 \\
Numbers & 44.7 & 55.3 \\
Hands & 57.8 & 42.2 \\
Lion & 9.7 & 90.3 \\
Rhinoceros & 57.5 & 42.5 \\
Camel & 18.3 & 81.7 \\
Digits Forward & 45.8 & 54.2 \\
Digits Backward & 30.3 & 69.7 \\
Sustained Attention & 16.9 & 83.1 \\
Subtraction 1 & 14.2 & 85.8 \\
Subtraction 2 & 51.9 & 48.1 \\
Subtraction 3 & 48.1 & 51.9 \\
Subtraction 4 & 51.7 & 48.3 \\
Subtraction 5 & 55.8 & 44.2 \\
Sentence 1 & 29.2 & 70.8 \\
Sentence 2 & 52.2 & 47.8 \\
Phonemic Fluency & 73.1 & 26.9 \\
Abstraction 1 & 35.8 & 64.2 \\
Abstraction 2 & 64.4 & 35.6 \\
Word 1 & 68.6 & 31.4 \\
Word 2 & 66.7 & 33.3 \\
Word 3 & 52.2 & 47.8 \\
Word 4 & 70.6 & 29.4 \\
Word 5 & 53.3 & 46.7 \\
Date & 26.4 & 73.6 \\
Month & 13.3 & 86.7 \\
Year & 19.4 & 97.2 \\
Day & 12.8 & 99.7 \\
Place & 3.3 & \\
City & 0.8 & \\
\hline \hline
\end{tabular}

Note. All values are expressed in percentage (\%). 
Table 3. Correlation coefficients of each item with total score and cognitive domains

\begin{tabular}{|c|c|c|c|c|c|c|c|}
\hline Item code & Total score & Mem & $\mathrm{EF}$ & VSS & Lang & ACWM & Ori \\
\hline TMT-B (adapted) & $.626^{* *}$ & $.426^{* *}$ & $.674^{* *}$ & $.544^{* *}$ & $.384^{* *}$ & $.447^{* *}$ & $.419^{* *}$ \\
\hline Cube & $.505^{* *}$ & $.221^{* * *}$ & $.491^{* *}$ & $.734^{* *}$ & $.348^{* *}$ & $.425^{* *}$ & $.183^{* *}$ \\
\hline Contour & $.257^{* *}$ & $.141^{* * *}$ & $.139^{* * *}$ & $.249^{* *}$ & $.168^{* *}$ & $.216^{* *}$ & $.266^{* *}$ \\
\hline Numbers & $.654^{* *}$ & $.354^{* *}$ & $.555^{* *}$ & $.797^{* *}$ & $.448^{* *}$ & $.465^{* *}$ & $.454^{* *}$ \\
\hline Hands & $.581^{* *}$ & $.281^{* *}$ & $.507^{* *}$ & $.798^{* *}$ & $.382^{* *}$ & $.423^{* *}$ & $.353^{* *}$ \\
\hline Lion & $.344^{* * *}$ & .102 & $.257^{* *}$ & $.283^{* *}$ & $.533^{* *}$ & $.178^{* *}$ & $.275^{* *}$ \\
\hline Rhinoceros & $.402^{* *}$ & $.165^{* *}$ & $.326^{* *}$ & $.301^{* *}$ & $.682^{* *}$ & $.258^{* *}$ & $.213^{* *}$ \\
\hline Camel & $.545^{* *}$ & $.310^{* *}$ & $.394^{* *}$ & $.347^{* *}$ & $.672^{* *}$ & $.385^{* *}$ & $.373^{* *}$ \\
\hline Digits Forward & $.470^{* *}$ & $.214^{* *}$ & $.308^{* * *}$ & $.306^{* *}$ & $.397^{* *}$ & $.606^{* *}$ & $.258^{* *}$ \\
\hline Digits Backward & $.396^{* *}$ & $.199^{* * *}$ & $.272^{* * *}$ & $.250^{* *}$ & $.215^{* *}$ & $.579^{* *}$ & $.193^{* *}$ \\
\hline Sustained Attention & $.580^{* *}$ & $.369^{* *}$ & $.329^{* *}$ & $.366^{* *}$ & $.380^{* *}$ & $.647^{* *}$ & $.483^{* *}$ \\
\hline Subtraction 1 & $.498^{* *}$ & $.238^{* * *}$ & $.379^{* * *}$ & $.357^{* *}$ & $.328^{* *}$ & $.604^{* *}$ & $.375^{* *}$ \\
\hline Subtraction 2 & $.536^{* *}$ & $.343^{* *}$ & $.395^{* *}$ & $.424^{* *}$ & $.369^{* *}$ & $.570 * *$ & $.324^{* *}$ \\
\hline Subtraction 3 & $.513^{* *}$ & $.281^{* *}$ & $.327^{* *}$ & $.416^{* *}$ & $.319^{* *}$ & $.629^{* *}$ & $.331^{* *}$ \\
\hline Subtraction 4 & $.485^{* *}$ & $.286^{* *}$ & $.332^{* *}$ & $.357^{* *}$ & $.296^{* *}$ & $.612^{* *}$ & $.277^{* *}$ \\
\hline Subtraction 5 & $.523^{* *}$ & $.250^{* * *}$ & $.371^{* * *}$ & $.445^{* *}$ & $.357^{* *}$ & $.632^{* *}$ & $.302^{* *}$ \\
\hline Sentence 1 & $.535^{* *}$ & $.280^{* *}$ & $.365^{* *}$ & $.364^{* *}$ & $.624^{* *}$ & $.423^{* *}$ & $.391^{* *}$ \\
\hline Sentence 2 & $.440^{* *}$ & $.228^{* * *}$ & $.274^{* *}$ & $.272^{* *}$ & $.607^{* *}$ & $.365^{* *}$ & $.267^{* *}$ \\
\hline Phonemic Fluency & $.449^{* *}$ & $.208^{* * *}$ & $.659^{* * *}$ & $.388^{* *}$ & $.338^{* *}$ & $.316^{* *}$ & $.206^{* *}$ \\
\hline Abstraction 1 & $.467^{* *}$ & $.216^{* * *}$ & $.635^{* *}$ & $.399^{* *}$ & $.351^{* *}$ & $.349^{* *}$ & $.248^{* *}$ \\
\hline Abstraction 2 & $.504^{* *}$ & $.274^{* *}$ & $.723^{* *}$ & $.440^{* *}$ & $.338^{* *}$ & $.340^{* *}$ & $.259^{* *}$ \\
\hline Word 1 & $.472^{* *}$ & $.671^{* *}$ & $.311^{* *}$ & $.238^{* *}$ & $.249^{* *}$ & $.242^{* *}$ & $.323^{* *}$ \\
\hline Word 2 & $.503^{* *}$ & $.740^{* *}$ & $.284^{* * *}$ & $.262^{* *}$ & $.252^{* *}$ & $.278^{* *}$ & $.341^{* *}$ \\
\hline Word 3 & $.612^{* *}$ & $.756^{* * *}$ & $.407^{* *}$ & $.365^{* *}$ & $.307^{* *}$ & $.398^{* *}$ & $.445^{* *}$ \\
\hline Word 4 & $.418^{* * *}$ & $.687^{* *}$ & $.194^{* * *}$ & $.183^{* *}$ & $.204^{* *}$ & $.227^{* *}$ & $.314^{* *}$ \\
\hline Word 5 & $.584^{* * *}$ & $.797^{* * *}$ & $.334^{* * *}$ & $.302^{* *}$ & $.296^{* *}$ & $.392^{* *}$ & $.442^{* *}$ \\
\hline Date & $.642^{* *}$ & $.473^{* *}$ & $.367^{* *}$ & $.394^{* *}$ & $.393^{* *}$ & $.456^{* *}$ & $.820^{* * *}$ \\
\hline Month & $.570^{* * *}$ & $.382^{* * *}$ & $.300^{* * *}$ & $.319^{* *}$ & $.385^{* *}$ & $.390^{* *}$ & $.797^{* *}$ \\
\hline Year & $.673^{* *}$ & $.462^{* * *}$ & $.400^{* * *}$ & $.424^{* *}$ & $.473^{* *}$ & $.452^{* *}$ & $.856^{* *}$ \\
\hline Day & $.552^{* *}$ & $.370^{* * *}$ & $.316^{* *}$ & $.319^{* *}$ & $.345^{* *}$ & $.391^{* *}$ & $.763^{* *}$ \\
\hline Place & $.277^{* *}$ & $.165^{* *}$ & $.136^{* * *}$ & $.157^{* *}$ & $.142^{* *}$ & $.198^{* *}$ & $.460^{* *}$ \\
\hline City & $.168^{* *}$ & .099 & .073 & .052 & $.163^{* *}$ & $.111^{*}$ & $.267^{* *}$ \\
\hline
\end{tabular}

Note. $\mathrm{Mem}=$ short-term memory $\mathrm{EF}=$ executive functions; $\mathrm{VSS}=$ visuospatial skills; Lang = language $; \mathrm{ACWM}=$ attention, concentration, and working memory; Ori $=$ orientation to time and space.

${ }^{*} p<.05 ;{ }^{* *} p<.001$.

(greater than 90\%) which suggests a low index of difficulty. On the other hand, Phonemic Fluency is the item with the lower hit rate $(26.9 \%)$.

The correlations between each item and the total score, and each item and the cognitive domains, as were conceptualized by the authors (Nasreddine et al., 2005), were also explored. As demonstrated in Table 3, each of the 32 items showed a significant $(p<.001)$ and positive correlation with the total score of the scale, with lower correlations of Contour, Lion, Place, and City items (respectively, .168, .344, .277, .257). These were simultaneously the items with higher hit rate (respectively, 97.8, 90.3, 96.7, 99.2). Therefore, these items reveal a lower contribution for the individual information obtained with the MoCA. Regarding the correlation coefficients of each item with the cognitive domains, all the items showed a significantly higher correlation with the respective domain, according to the structure proposed by the authors, as compared to the correlations with any other domain. With the exception of the Contour, Place, and City items, that showed lower correlations with any domain. Although the Contour did not exhibit a differentiated correlation with any domain, the Place and City showed a significantly higher correlation with Orientation than with any of the others domains. Despite the consideration that the Phonemic Fluency contributes to the two domains in the original MoCA formulation, we found a higher correlation with the Executive Functions domain than with the Language domain.

As shown in Table 4, correlations between each cognitive domain and the MoCA total score were high and positive, ranging from .711 to .801 . These correlations are suggestive of construct related validity. Furthermore, we can observe that each domain showed significantly higher correlation with the MoCA total score than with another domain, what is suggestive of discriminative power of domains.

\section{Scale Reliability}

Internal consistency reliability of the MoCA was estimated using Cronbach's $\alpha$. In the total paired sample $(n=360)$, we found a Cronbach's $\alpha$ of .903 that confirms the overall 
Table 4. Correlation coefficients of the cognitive domains and total score

\begin{tabular}{lccccccc}
\hline \hline & Total score & EF & VSS & Lang & Mem & ACWM Ori \\
\hline Total score & - & & & & & & \\
EF & .757 & - & & & & & \\
VSS & .752 & .655 & - & & & & \\
Lang & .767 & .638 & .549 & - & & & \\
Mem & .711 & .418 & .372 & .369 & - & & \\
ACWM & .801 & .537 & .569 & .552 & .427 & - & \\
Ori & .745 & .420 & .442 & .478 & .513 & .515 & - \\
\hline \hline
\end{tabular}

Note. All correlation coefficient were significant at the .001 level. $\mathrm{EF}=$ executive functions; VSS = visuospatial skills; Lang = language; $\mathrm{Mem}=$ short-term memory; $\mathrm{ACWM}=$ attention, concentration, and working memory; $\mathrm{Ori}=$ orientation to time and space.

reliability of the scale when used to examine Portuguese subjects. A more detailed analysis reveals that there is no improvement regarding the reliability coefficient value with the exclusion of any item of the scale. This reliability coefficient was also computed for each sub-group. In the clinical group $(n=180)$ the respective value was .883 , while in the control group $(n=180)$ it was .678 . In the total sample $(n=830)$ we found a Cronbach's $\alpha$ of .905 .

\section{Diagnostic Accuracy}

The ROC curve analysis was conducted on paired sample $(n=360)$ to evaluate the diagnostic accuracy of MoCA to discriminate MCI and AD patients from cognitively healthy adult. The discriminant potential of the MoCA for MCI was high, with an AUC of .856 (95\% confidence interval $[\mathrm{CI}]=.796-.904)$ and for AD was excellent, with an AUC of .980 (95\% CI = .947-.995). According to the Youden index, the optimal cutoff point for MCI was below 22 points (sensitivity $=81 \%$; specificity $=77 \%$ ). For the AD patients, the optimal cutoff point was below 17 points (sensitivity $=88 \%$; specificity $=98 \%$ ).

\section{CONFIRMATORY FACTOR ANALYSIS}

Model Specification

The CFA was performed to provide further evidence of the MoCA's construct validity. Three models were contemplated in the analyses. The first model (a six-factor model) was based on the original conceptual model proposed by the MoCA's authors (Nasreddine et al., 2005). The second model (two-factor model) matched the two factor model proposed by Duro et al. (2010). Finally, we included a third model (one-factor second-order model) which considers that all the first-order factors are contributing to a common underlying second-order factor that we named "Cognition." With this latter model, we intend to explore the unidimensionality tendency of the MoCA, similarly to what was found in some studies with other cognitive screening tests, as MMSE (e.g., Jones \& Gallo, 2000).

Model Assessment

To determine the best model, the three models were tested using the CFA and the model fit statistics compared. The fit statistics for each model, in total sample $(n=830)$, are summarized in Table 5.

We can observe that the different fit models indices reflect good fit of the three models. All three models had a relative $\chi^{2}$ close 2 , the CFI and the TLI were above .95 , and an RMSEA was less than .05. However, the six-factor model theoretically proposed by the authors showed a general better fit to the observed data in all the indices considered. The improved fit of six-factor model was significantly higher than the two-factor model in this study sample $\left(\Delta \chi^{2}\right.$ $(15)=269.165 ; p<.001)$ and than the one-factor secondorder model $\left(\Delta \chi^{2}(9)=128.703 ; p<.001\right)$. The standardized factor weights and the item's squared multiple correlations of the six-factor model are compiled in Table 6. General factor weights and fit indices are suggestive of the MoCA six-factor factorial related validity.

Following the approach of Fornell and Larcker (1981), the composite reliability indices were computed for each latent factor. We found a value of .74 for Executive Function; .84 for Language; .89 for Visuospatial Skills; .87 for Short-term Memory; .91 for Attention, Concentration, and Working Memory; and .95 for Orientation, which is indicative of good convergent related validity for each factor.

Given the fit results for the six-factor model we also examined the convergent and discriminant validity of the six factors using a stringent procedure outlined by Fornell and Larcker (1981). Regarding convergent validity, we computed the averaged variance extracted (AVE) which denotes the proportion of variance in the items explained by the underlying factor. The respective results range from .46 to .75, which is suggestive of appropriated convergent validity, according the criterion AVE >.5 (Fornell \& Larcker, 1981). The discriminant validity of the factors was measured by comparing the AVE of each factor with the square of correlation between the factors. According to Fornell and Larcker (1981),

Table 5. it indices of the Confirmatory Factor Analysis models

\begin{tabular}{|c|c|c|c|c|c|c|c|}
\hline Models & $\chi^{2}$ & $d f$ & $p$ & $\chi^{2} / d f$ & CFI & TLI & RMSEA \\
\hline Six-factor model & 708.877 & 448 & $<.001$ & 1.582 & .981 & .978 & .026 \\
\hline Two-factor model & 1045.867 & 463 & $<.001$ & 2.259 & .956 & .953 & .039 \\
\hline One-factor model (second-order) & 872.094 & 457 & $<.001$ & 1.908 & .969 & .966 & .033 \\
\hline
\end{tabular}

$\chi^{2}=$ Chi-square test statistic; $d f=$ degrees of freedom; $\chi^{2} / d f=$ relative Chi-square; CFI=Comparative Fit Index; TLI=Tucker-Lewis Index; RMSEA $=$ root mean square error of approximation. 
Table 6. Standardized regression weights and squared multiple correlations for six-factor Model

\begin{tabular}{|c|c|c|}
\hline Latent and observed variables & Standardized regression weights & $R^{2}$ \\
\hline \multicolumn{3}{|l|}{ Factor 1: Executive functions } \\
\hline TMT-B (adapted) & .89 & .79 \\
\hline Phonemic Fluency & .21 & .04 \\
\hline Abstraction 1 & .66 & .44 \\
\hline Abstraction 2 & .75 & .56 \\
\hline \multicolumn{3}{|l|}{ Factor 2: Language } \\
\hline Lion & .71 & .51 \\
\hline Rhinoceros & .67 & .45 \\
\hline Camel & .84 & .71 \\
\hline Sentence 1 & .70 & .49 \\
\hline Sentence 2 & .60 & .36 \\
\hline Phonemic Fluency & .55 & .30 \\
\hline \multicolumn{3}{|l|}{ Factor 3: Visuospatial skills } \\
\hline Cube & .79 & .63 \\
\hline Contour & .84 & .70 \\
\hline Numbers & .83 & .69 \\
\hline Hands & .82 & .67 \\
\hline \multicolumn{3}{|l|}{ Factor 4: Short-term memory } \\
\hline Word 1 & .72 & .51 \\
\hline Word 2 & .75 & .56 \\
\hline Word 3 & .83 & .69 \\
\hline Word 4 & .65 & .42 \\
\hline Word 5 & .83 & .68 \\
\hline \multicolumn{3}{|c|}{ Factor 5: Attention, concentration, and working memory } \\
\hline Digits Forward & .61 & .37 \\
\hline Digits Backward & .53 & .28 \\
\hline Sustained Attention & .94 & .89 \\
\hline Subtraction 1 & .73 & .53 \\
\hline Subtraction 2 & .69 & .48 \\
\hline Subtraction 3 & .78 & .62 \\
\hline Subtraction 4 & .79 & .62 \\
\hline Subtraction 5 & .80 & .63 \\
\hline \multicolumn{3}{|c|}{ Factor 6: Temporal and spatial orientation } \\
\hline Date & .91 & .83 \\
\hline Month & .90 & .82 \\
\hline Year & .98 & .95 \\
\hline Day & .91 & .83 \\
\hline Place & .80 & .64 \\
\hline City & .65 & .43 \\
\hline
\end{tabular}

two factors showed discriminant validity when the AVE is greater than the square of correlation between the factors. Except for Executive Functions and Language, which share the variance of the Phonemic Fluency, we have found discriminant validity between the factors.

\section{DISCUSSION}

The overall aim of the present study was to evaluate the factorial structure of the MoCA and analyze its construct related validity in cognitively healthy subjects, as well as in the MCI and the AD patients. To date, there were no other research studies of the factorial structure of the MoCA in community samples, and only one analyzed the dimensions underlying the MoCA in a heterogeneous clinical sample with cognitive decline. Thus, this study contributes to overcome a significant gap in the evaluation of the construct related validity of this instrument.

To provide further evidence to MoCA's construct related validity, several models were tested using CFA: a six-factor model based on the conceptual model proposed by the authors of the MoCA (Nasreddine et al., 2005); a two-factor model proposed by Duro et al. (2010); and a one-factor secondorder model with all the first-order factors contributing to a common second-order factor "Cognition." Although all models showed a good fit in our data, the six-factor model showed the better absolute and relative fit indices, proving to have a significantly better fit than the others factorial models evaluated. Thus, this study corroborates the six-dimensional structure of the MoCA proposed by the authors. This six-dimensional 
approach was confirmed, not only by the improved fit of the model, but also by the validity analysis of the test where we were able to observe stronger intercorrelations between each item and the cognitive domains and between each cognitive domain and the MoCA total score. However, we found a lower contribution of the Phonemic Fluency task for the assessment of the Executive Function domain, so it is advisable to caution its interpretation in this sense.

The item analysis reveals an overall psychometric adequacy of the items of the MoCA. However, some problems were identified regarding the items Contour, Place, and City, which showed a higher hit rate and consequently lower correlations with any cognitive domain and with the total score. However, these items did not compromise the overall six-factor model fit, showing an appropriated factor weights in model. The MoCA has also shown good reliability, either through the Cronbach's $\alpha$ or through the composite reliability indices.

Regarding to the MoCA's diagnostic accuracy to discriminate MCI and AD patients from cognitively healthy adults, with an optimal cutoff point below 22 points for the MCI patients, the MoCA showed a good sensitivity (81\%) and specificity (77\%). Likewise, with an optimal cutoff point below 17 points for the AD patients, the MoCA showed an excellent sensitivity (88\%) and specificity (98\%).

Our results allowed to establish the factorial, convergent, and discriminant validity of the six-dimensional structure of the MoCA, and then proving its construct related validity. These findings permitted the calculation of performance profiles based on the results of the six cognitive dimensions. The possibility of a more comprehensive analysis of the performance in a brief cognitive screening test is a valuable advantage, resulting in an important contribution to outline a more systematic and comprehensive neuropsychological evaluation. Although the six-factor model shows the best fit, our findings allow to additionally support a second-order factor model, which considers that all the first-order factors are contributing to a common underlying second-order factor: "Cognition." This reveals a second-order unidimensional tendency, and serves as a good indicator that the MoCA, as a whole, evaluates individuals' global cognition (a positive finding considering that the MoCA is a brief cognitive screening instrument). The authors (Nasreddine et al., 2005) $a$ priori hypothesized dimensions are consistent with the best MoCA's factor structure of this study. Indeed, this study provides additional evidence for the MoCA multifactorial nature, supporting the idea that this screening test does not measure only a global cognitive ability (total score) but the sub-scores extracted reflect different constructs or specific aspects of cognitive functioning, offering an empirical rational for examining separate indexes scores in the context of a profile analysis. So, the MoCA interpretation could also examine the relative strengths and weaknesses (or higher and lower scores) for further additional hypothesis testing.

The current study has some limitations regarding the clinical samples size which did not allow a more detailed analysis of the MoCA's structure in these groups. Some future considerations should be taken into account when analyzing the present results, namely the need for more studies conducted in different cultural contexts other than the Portuguese, as well as for different diagnostic groups to further confirm the proposed six-dimensional structure underlying the MoCA and extend the present findings. Furthermore, it will be interesting to analyze the invariance of factorial structure across different groups, like diagnostic groups, age groups, and educational groups.

In summary, the MoCA has proved to be an appropriate measure for brief cognitive screening taking into account different cognitive domains such as: short-term memory; executive functions; visuospatial abilities; language; attention, concentration, working memory; and temporal and spatial orientation. These constructs have demonstrated to be structurally related. The present findings establish the factorial, convergent, and discriminant validity, providing good evidence of the construct related validity of the MoCA, and will enable clinicians and others researchers to use this test, including the total score and also mainly their six latent dimensions to achieve a better understanding of the individuals' cognitive profile.

\section{ACKNOWLEDGMENTS}

This work was supported by the Fundação para a Ciência e Tecnologia [Portuguese Foundation for Science and Technology], (S.F., SFRH/BD/ 38019/2007); and by the Fundação Calouste Gulbenkian [Calouste Gulbenkian Foundation], (Proc.74569, SDH-22 Neurociências). All authors of this study declare that there are no conflicts of interest.

\section{REFERENCES}

Alves, L., Simões, M.R., \& Martins, C. (2009). Teste de Leitura de Palavras Irregulares (TeLPI) [Reading Word Irregular Test]. Coimbra, Portugal: Serviço de Avaliação Psicológica da Faculdade de Psicologia e de Ciências da Educação da Universidade de Coimbra [Psychological Assessment Department, Faculty of Psychology and Educational Sciences, University of Coimbra].

American Psychiatric Association. (2000). Diagnostic and Statistical Manual of Mental Disorders (4th ed. - Text Revised). Washington, DC: American Psychiatric Association Press.

Appels, B.A., \& Scherder, E. (2010). The diagnostic accuracy of dementia-screening instruments with an administration time of 10 to 45 minutes for use in secondary care: A systematic review. American Journal of Alzheimer's Disease and Other Dementias, 25(4), 301-316. doi:10.1177/1533317510367485

Barreto, J., Leuschner, A., Santos, F., \& Sobral, M. (2008). Escala de Depressão Geriátrica [Geriatric Depression Scale]. In Grupo de Estudos de Envelhecimento Cerebral e Demências [Study Group on Brain Aging and Dementia] (Ed.), Escalas e testes na demência [Scales and tests in dementia] (pp. 69-72). Lisbon: GEECD.

Byrne, B.M. (2010). Structural equation modeling with Amos: Basic concepts, applications, and programming (2nd ed.). New York: Routledge.

Duro, D., Simões, M.R., Ponciano, E., \& Santana, I. (2010). Validation studies of the Portuguese experimental version of Montreal Cognitive Assessment (MoCA): Confirmatory factor analysis. Journal of Neurology, 257(5), 728-734. doi:10.1007/ s00415-009-5399-5 
Folstein, M., Folstein, S., \& McHugh, P. (1975). Mini-Mental State: A practical method for grading the cognitive state of patients for the clinician. Journal of Psychiatric Research, 12(3), 189-198.

Fornell, C., \& Larcker, D.F. (1981). Evaluating SEM with unobserved variables and measurement error. Journal of Marketing Research, 18, 39-50.

Freitas, S., Santana, I., \& Simões, M.R. (2010). The sensitivity of the MoCA and MMSE to cognitive decline: A longitudinal study. Alzheimer's \& Dementia, 6(4), S353-S354.

Freitas, S., Simões, M.R., Alves, L., \& Santana, I. (2011). Montreal Cognitive Assessment (MoCA): Normative study for the Portuguese population. Journal of Clinical and Experimental Neuropsychology, doi:10.1080/13803395.2011.589374

Freitas, S., Simões, M.R., Martins, C., Vilar, M., \& Santana, I. (2010). Estudos de adaptação do Montreal Cognitive Assessment (MoCA) para a população portuguesa [Montreal Cognitive Assessment (MoCA): Portuguese adaptation studies]. Avaliação Psicológica, 9(3), 345-357.

Garret, C., Santos, F., Tracana, I., Barreto, J., Sobral, M., \& Fonseca, R. (2008). Avaliação Clínica da Demência [Clinical Dementia Rating Scale]. In Grupo de Estudos de Envelhecimento Cerebral e Demências [Study Group on Brain Aging and Dementia] (Ed.), Escalas e testes na demência [Scales and tests in dementia] (pp. 17-32). Lisbon: GEECD.

Ginó, S., Mendes, T., Ribeiro, F., Mendonça, A., Guerreiro, M., \& Garcia, C. (2008). Escala de Queixas de Memória [Memory Complaints Scale]. In Grupo de Estudos de Envelhecimento Cerebral e Demências [Study Group on Brain Aging and Dementia] (Ed.), Escalas e testes na demência [Scales and tests in dementia] (pp. 117-120). Lisbon: GEECD.

Guerreiro, M. (1998). Contributo da Neuropsicologia para o estudo das demências [Contribution of Neuropsychology to the study of dementia] (Unpublished doctoral dissertation). University of Lisbon, Lisbon.

Guerreiro, M., Fonseca, S., Barreto, J., \& Garcia, C. (2008). Escala de avaliação da Doença de Alzheimer [Alzheimer's disease assessment scale]. In Grupo de Estudos de Envelhecimento Cerebral e Demências [Study Group on Brain Aging and Dementia] (Ed.), Escalas e testes na demência [Scales and tests in dementia] (pp. 42-68). Lisbon: GEECD.

Hair, J.F., William, C.B., Barry, J.B., Rolph, E.A., \& Ronald, L.T. (2010). Multivariate data analysis (7th ed.). New Jersey: Pearson Education.

Himmelfarb, S. (1993). The measurement of attitudes. In A.H. Eagly \& S. Chaiken (Eds.), The psychology of attitudes (pp. 23-87). Orlando: Harcourt Brace Jovanovich Inc.

Hughes, C.P., Berg, L., Danziger, W.L., Coben, L.A., \& Martin, R.L. (1982). A new clinical scale for the staging of dementia. The British Journal of Psychiatry, 140, 566-572.

Ismail, Z., Rajji, T.K., \& Shulman, K.I. (2009). Brief cognitive screening instruments: An update. International Journal of Geriatric Psychiatry, 25(2), 111-120. doi:10.1002/gps.2306

Jacova, C., Kertesz, A., Blair, M., Fisk, J.D., \& Feldman, H.H. (2007). Neuropsychological testing and assessment for dementia. Alzheimer's Dementia, 3(4), 299-317. doi:10.1016/ j.jalz.2007.07.011

Jones, R.N., \& Gallo, J.J. (2000). Dimensions of the Mini-Mental State Examination among community dwelling older adults. Psychological Medicine, 30, 605-618.

Koski, L., Xie, H., \& Finch, L. (2009). Measuring cognition in a geriatric outpatients clinic: Rash analysis of the Montreal Cognitive Assessment. Journal of Geriatric Psychiatry and Neurology, 22(3), 151-160. doi:10.1177/0891988709332944

Koski, L., Xie, H., \& Konsztowicz, S. (2011). Improving precision in the quantification of cognition using the Montreal Cognitive Assessment and the Mini-Mental State Examination. International Psychogeriatrics, 1, 1-9. doi:10.1017/S1041610210002450

Lonie, J.A., Tierney, K.M., \& Ebmeier, K.P. (2009). Screening for mild cognitive impairment: A systematic review. International Journal of Geriatric Psychiatry, 24(9), 902-915. doi:10.1002/ gps. 2208

Marôco, J. (2010). Análise de equações estruturais: Fundamentos teóricos, software \& aplicações [Structural equations analysis: Theoretical foundations, software \& applications]. Pêro Pinheiro: ReportNumber.

McKhann, G., Drachman, D., Folstein, M., Katzman, R., Price, D., \& Stadlan, E.M. (1984). Clinical diagnosis of Alzheimer's disease: Report of the NINCDS-ADRDA Work Group under the auspices of the Department of Health and Human Services Task Force on Alzheimer's disease. Neurology, 34, 939-944.

Nasreddine, Z., Phillips, N.A., Bédirian, V., Charbonneau, S., Whitehead, V., Collin, I., ... Chertkow, H. (2005). The Montreal Cognitive Assessment, MoCA: A brief screening tool for Mild Cognitive Impairment. American Geriatrics Society, 53(4), 695-699. doi:10.1111/j.1532-5415.2005.53221.x

Petersen, R.C. (2004). Mild cognitive impairment as a diagnostic entity. Journal of Internal Medicine, 256(3), 183-194. doi:10.1111/j.1365-2796.2004.01388.x

Petersen, R.C. (2007). Mild cognitive impairment. Continuum: Lifelong Learning in Neurology, 13(2), (Dementia) 15-38.

Rosen, W.G., Mohs, R.C., \& Davis, K.L. (1984). A new rating scale for Alzheimer's Disease. American Journal of Psychiatry, 141, 1356-1364.

Schmand, B., Jonker, C., Hooijer, C., \& Lindeboom, J. (1996). Subjective memory complaints may announce dementia. Neurology, 46(1), 121-125.

Simões, M.R., Freitas, S., Santana, I., Firmino, H., Martins, C., Nasreddine, Z., \& Vilar, M. (2008). Montreal Cognitive Assessment (MoCA): Versão portuguesa [Montreal Cognitive Assessment (MoCA): Portuguese version]. Coimbra, Portugal: Serviço de Avaliação Psicológica da Faculdade de Psicologia e de Ciências da Educação da Universidade de Coimbra [Psychological Assessment Department, Faculty of Psychology and Educational Sciences, University of Coimbra].

Yesavage, J.A., Brink, T.L., Rose, T.L., Lum, O., Huang, V., Adey, M., \& Leirer, V.O. (1983). Development and validation of a geriatric depression screening scale: A preliminary report. Journal of Psychiatric Research, 17(1), 37-49. 